\title{
Derechos humanos en el aire: Una propuesta educativa en Derechos Humanos y habilidades comunicativas orientada a niños y jóvenes vulnerables utilizando la radio
}

\begin{abstract}
Human Rights on Air: A educational proposal in Human Rights and communication abilities towards children and adolescents in the most vulnerable populations by working through radio.
\end{abstract}

\author{
Patricia Cocq Muñoz \\ Unidad de Educación y Promoción del Instituto Nacional de Derechos Humanos. \\ pacocq@uc.cl
}
Resumen El presente artículo aborda los referentes teóricos para la elaboración de una propuesta de taller de radio desarrollado desde una perspectiva de educación en Derechos Humanos, orientado a niños, niñas y jóvenes de sectores sociales vulnerables, además de los aprendizajes obtenidos de la experiencia de un taller real que pretendía reforzar el derecho a la participación de un grupo de jóvenes de la comuna de La Pintana. Además, resume el análisis de tres experiencias de radio y Derechos Humanos en Latinoamérica, que son insumos para la construcción de una propuesta.

\begin{abstract}
This article addresses the theoretical references to elaborate a proposal for a radio workshop with a human rights education's perspective, orientated to children and young people in socially vulnerable sectors, as well as the acquired lessons of the experience of a real workshop that was implemented to enhance the right to participation of a group of young people from La Pintana district. In addition, it summarizes the analysis of three radio and human rights experiences in Latin America that are inputs for the elaboration of a proposal.
\end{abstract}
Palabras Radio, derechos humanos, educación en derechos humanos, comunicación y educación, radio y clave niñez.
Key Radio, Human Rights, Human Rights Education, Communication and Education, Radio and words Childhood.

- Agradecimientos: Esta investigación contó con el apoyo de la Facultad de Comunicaciones de la Universidad Católica de Chile, Puentes UC y la ODP de la Municipalidad de la Pintana, Chile. 


\section{Presentación}

El problema original del cual surge la propuesta, responde a una necesidad de la Oficina de Protección de Derechos de la Infancia de la comuna de La Pintana, entidad que presentaba como parte de su plan de trabajo un eje relacionado con la promoción de los derechos de niñas, niños y adolescentes (NNA de ahora en adelante) a través de su protagonismo, recogiendo sus opiniones y generando oportunidades de participación. Para lograr este objetivo, solicitaron a la Facultad de Comunicaciones ${ }^{1}$ de la Pontificia Universidad Católica el diseño y desarrollo de un taller de comunicaciones para jóvenes reporteros y reporteras del sector San Ricardo de La Pintana, que se llevó a cabo en conjunto con el Programa Puentes UC.

El taller, planificado por el equipo integrado por académicas y estudiantes de posgrado², y ejecutado por estos últimos, se realizó entre mayo y septiembre del 2012, con un cierre más bien simbólico, ya que no se logró llegar a los productos esperados, que eran piezas comunicacionales audiovisuales. Luego de desarrollar esta experiencia, el equipo de trabajo en terreno se cuestionó los alcances de la misma, ya que se presentaron situaciones no esperadas, como la dificultad de implementar los contenidos propuestos inicialmente, el manejo de grupo, el enfoque de derechos y el carácter participativo que se le quiso imprimir y que era el desafío principal.

En una sistematización de esta experiencia, se plantea que algunos de los aprendizajes fueron: la oportunidad de derribar prejuicios al usar las entrevistas como una posibilidad de conocer realidades diversas; el diálogo intergeneracional; conocer los medios de comunicación y su dinámica de trabajo; mirar críticamente su contexto y proponer transformaciones; expresarse frente a sus pares, superando el miedo al ridículo y dialogar para tomar acuerdos colectivos (Gómez Magaña, 2013).

Todos los logros identificados son valiosos desde el punto de vista del aprendizaje y el desarrollo social de las y los niños participantes. Sin embargo, si se revisa el mandato que motivó la experiencia, de potenciar el derecho a la participación como derecho de NNA y

1 El equipo fue integrado por Felipe Gómez Magaña y Patricia Coca Muñoz, estudiantes del Magíster en Comunicación Social con Mención en Comunicación y Educación, las docentes Rayén Condeza y Myrna Gálvez. Además, participaron Claudia Trevizán, de la OPD de La Pintana, Patricia Contreras y equipo del PPC (Programa de Prevención Comunitaria) Acuarela, a cargo de Comunidad Papa Juan XXIII.

2 Este proyecto fue la base de dos tesis de posgrado realizadas por Patricia Coca Muñoz (cuya profesora guía fue Myrna Gálvez) y Felipe Gómez Magaña (guiada por Rayén Condeza), detalladas en la bibliografía, 
de capacitar en herramientas de creación de contenido, el proyecto no cumplió con esas expectativas, teniendo logros más bien tangenciales, aunque importantes.

De esta forma, ya propósito del análisis del Taller de Reporteros/as, se genera la oportunidad de diseñar una propuesta de trabajo que recoja los aciertos, pero que también abarque estas otras dimensiones que no se concretaron, pero que siguen siendo desafíos de la OPD La Pintana, como es la educación en Derechos Humanos para niños, niñas y jóvenes en contextos de vulnerabilidad. El desafío del Taller "Derechos humanos en el aire" fue empoderar a los adolescentes en sus derechos utilizando la radio, desde un enfoque integral de Derechos Humanos (Cocq Muñoz, 2014).

\section{Referentes teóricos}

Este proyecto se enfoca en un público concreto y específico: niños, niñas y jóvenes de sectores vulnerables, espacios donde la dimensión del trabajo con Derechos Humanos y medios de comunicación es relevante y necesario. Este trabajo busca responder a cómo se genera la participación de los niños como sujetos de derechos, dados en situación de pobreza.

La niñez es un concepto propio de la modernidad que intenta universalizar una síntesis de aspectos propios de su conceptualización. No obstante, se adoptará un concepto de niñez vinculado a sus raíces culturales y significativas, ya que constituyen elementos de identidad y diferencia, de construcción subjetiva, de experiencia y de dotación de vida. Desde esta perspectiva entenderemos a niños y niñas como un otro diferente, específico, que de acuerdo a la compleja red de interacciones particulares de su entorno se construye cotidianamente, pero en una construcción desde su particularidad de persona, asumiendo su propia personalidad, su identidad constitutiva.

En este contexto, la niñez es el producto de un imaginario social concreto habitado por las concepciones sociales imperantes. El proyecto asume esa diversidad cultural y la integra en sus procesos.

Por su parte, los Derechos Humanos proporcionan un marco conceptual adaptativo para entender la infancia desde su especificidad cultural. Basta considerar la Convención Internacional de los Derechos del Niño, que permite trabajar su noción en una realidad particular, considerando a niñas y niños como sujetos de derecho, es decir, como personas 
dadas y no como personas en construcción hacia un "alguien". Esta Convención no solo habla de protección a la niñez (noción clásica) sino que establece una normativa internacional que protege, garantiza y promueve derechos.

La participación de niños y niñas se entiende como "el ejercicio del poder que tienen los niños y las niñas para hacer que sus opiniones sean tomadas en cuenta seriamente y para asumir en forma responsable, según su grado de madurez y desarrollo, decisiones compartidas con otros en asuntos que afectan sus vidas y la de su comunidad" (Nomura y Solari, 2005). Esta concepción asume un enfoque inclusivo y decidor de NNA en la construcción de su entorno, que debe ser tomado en cuenta por las autoridades en tanto otros diferentes que participan en la construcción democrática.

Los Derechos Humanos, además, nos permiten interpretar la pobreza y la exclusión social tanto para enfrentarla como un problema de justicia social, centrada ya no solo en el individuo sino en la estructura social. "La pobreza es, al mismo tiempo causa y consecuencia de las violaciones de los Derechos Humanos: causa porque las personas pobres permanecen invisibles y, así, alejadas de los intentos de ayudarles a reclamar sus derechos, y porque las manifestaciones de la pobreza son el hambre, la carencia de hogar y el analfabetismo, entre otras muchas; consecuencia porque la pobreza puede derivarse de una acción u omisión, es decir, una violación de un Derecho Humano, como por ejemplo, la ausencia de acceso a los recursos sanitarios básicos o el desalojo forzado" (Formisano, 2011).

A la carencia de recursos materiales y de acceso a servicios o derechos sociales necesarios para una vida digna, el economista Amartya Sen (Sen, 2000) agrega el enfoque de capacidades, señalando que la pobreza también se refiere a la imposibilidad de desarrollar las capacidades que generan un mayor desarrollo humano y estimulan la participación social. “Desde esta perspectiva, la pobreza debe concebirse como la privación de capacidades básicas y no meramente como la falta de ingresos, que es el criterio habitual con el que se identifica la pobreza". Para Amartya Sen las "capacidades" comprenden todo aquello que permiten a una persona estar bien nutrido/a, escribir, leer y comunicarse, tomar parte de la vida comunitaria; formar parte de estas "capacidades". Es importante destacar que Sen incluye en estas capacidades las habilidades comunicativas.

De esta forma, los medios de comunicación operados por los/as protagonistas de estos sectores, son un espacio tanto de participación como de reivindicación de Derechos Humanos. Pero ¿cómo enseñar los Derechos Humanos más allá de una enunciación de ellos? 


\section{Educación en Derechos Humanos}

En la defensa de los Derechos Humanos el principal garante es el Estado, que se compromete a través de tratados internacionales, a respetar, proteger y cumplir los derechos de todas las personas que viven en su territorio (Oficina en México del Alto Comisionado de las Naciones Unidas para los Derechos Humanos, OACNUDH, 2011). Aun así, la sociedad civil tiene un rol preponderante en la denuncia y control de las acciones del Estado en esta materia. Es por eso que no solo basta la existencia del catálogo de derechos, sino que el conocimiento de ellos y de las estrategias para su exigibilidad se tornan en una urgencia para avanzar a una sociedad más justa y que respete la dignidad de las personas.

La educación se presenta como un instrumento principal para promover y facilitar el ejercicio de los derechos (Instituto Nacional de Derechos Humanos, 2012). Esta disciplina o ámbito es conocido como educación en Derechos Humanos, la que puede definirse como "un conjunto de actividades de educación, capacitación y difusión de información orientadas a crear una cultura universal de los Derechos Humanos"(Naciones Unidas, 2006).

La educación en Derechos Humanos es, en sí misma, un derecho centrado en poseer, buscar y recibir información sobre todos los derechos, tanto en la educación formal, la no formal y la informal (Naciones Unidas, 2012). Es importante considerar que los Derechos Humanos no se remiten solo a normas, también son un modelo ético que puede orientar la relación entre las personas y se basa en los valores de la igualdad, justicia y verdad. Estos derechos constituyen una tarea constante en la cual trabajar, la que no se agota en acciones aisladas, sino que se requiere la participación de todos los sectores de la sociedad (Instituto Nacional de Derechos Humanos, 2012). Tomando en cuenta esto, se puede hablar de educación sobre los Derechos Humanos, que contempla conocer y comprender las normas y principios y los mecanismos para su exigibilidad; educación por medio de los Derechos Humanos, que se genera en un proceso respetuoso de los derechos de las personas involucradas en el proceso educativo; y la educación para los Derechos Humanos, que forma a las personas para que disfruten sus derechos, los ejerzan, y respeten los de los demás, convirtiéndose a su vez en defensores y defensoras (Naciones Unidas, 2012).

Desde un punto de vista práctico, el integrar los Derechos Humanos en la práctica educativa de un taller de radio, es compatible con otro tipo de contenidos, ya que invita 
a hacer cambios en las prácticas habituales, y a incorporar una mirada de Derechos Humanos en todo el quehacer y no solo como un referente temático.

\section{Un modelo para educar en Derechos Humanos}

Cuando se planifica una intervención educativa de este tipo, es necesario considerar profundamente las circunstancias y características de la comunidad donde será realizada (Tibitts, s/f). Para esto, hay que definir cuál será el modelo de educación en Derechos Humanos que será utilizada, contándose para ello con tres posibles modelos: el modelo de valores y percepción, el modelo de responsabilidad y el modelo de transformación.

Para diseñar los contenidos del taller se analizaron los tres modelos propuestos por Tibitts (s/f) y sus principales características, para ahondar sobre uno de ellos. A partir de la investigación de diversos programas de Derechos Humanos, Tibbits propone tres modelos idealizados, vinculados con grupos objetivos y con distintas estrategias para el cambio social. Estos modelos no están pensados como pautas a seguir, sino como bases para pensar las intervenciones educativas.

Los tres modelos propuestos por Tibbitts son:

Modelo de valores y percepción: cuyo objetivo es transmitir un concepto básico de Derechos Humanos a la opinión pública.

Modelo de responsabilidad: se orienta a una audiencia de la que se espera asuma responsabilidad en la defensa de los derechos desde sus funciones profesionales y laborales.

Modelo de transformación: en este modelo, el objetivo es educar para que la persona identifique las vulneraciones de Derechos Humanos y se comprometa a prevenirlos. El grupo objetivo no solo es la persona, sino también la comunidad, ya que se requiere interacción y colaboración de sus integrantes.

Es este último el que se utilizó para la propuesta. Un aspecto importante en este modelo es que se dirige a personas que viven vulneración de sus derechos y que tienen interés en promover los Derechos Humanos activamente. Algunos de los grupos con que se trabaja desde este enfoque son: refugiados/as, migrantes, mujeres víctimas de violencia, niños y niñas, personas que viven en sectores de pobreza, diversidades sexuales y otros grupos que 
han sufrido discriminación. Este modelo interesa en su aplicación ya que trabaja con NNA vulnerables en sus derechos y permite desarrollar las capacidades necesarias para prevenir y hacer respetar los Derechos Humanos en un espacio habitado por lo comunitario.

\section{Del derecho a participar a la cultura participativa}

Uno de los desafíos de cualquier proceso comunicativo y, más aún, educomunicativo es generar una metodología participativa. Es decir, no basta solo con conocer los derechos, sino que es importante ejercerlos y a través de la radio, en este caso, ejercemos uno fundamental. ¿Pero qué se entiende cuando se habla de participación? Es tan relevante la idea de la participación, que se establece como un Derecho Humano, del cual se abordará especialmente el referido a NNA, quienes son la audiencia especifica de la propuesta de este trabajo.

Niños y niñas son reconocidos como sujetos de derechos desde hace poco tiempo, en comparación con la historia de la Humanidad. La necesidad de entregarles una protección especial para el goce de sus derechos, tuvo su primera afirmación en la Declaración de Ginebra de 1924 sobre los Derechos del Niño y en la Declaración de los Derechos del Niño adoptada por la Asamblea General de Naciones Unidas el 20 de noviembre de 1959.

Dentro de los esfuerzos por establecer niveles y tipos de participación, se puede citar la "escalera" de Roger Haart (1993), modelo que sirve para establecer estrategias de participación desde el nivel simbólico hasta el nivel de una participación auténtica. En este esquema se busca establecer niveles de participación dependiendo de factores como la iniciativa que genera el proceso, quiénes se hacen cargo de guiarlos y la forma de tomar las decisiones, además del protagonismo infantil y/o de las personas adultas.

Dentro de este modelo, se considera como elemento importante la edad de niños y niñas que participan de las iniciativas. Así, a mayor edad será menor la participación de personas adultas y viceversa, lo que tiene relación con la idea de progresividad de los derechos de niños y niñas, es decir, que a medida que se desarrollan en distintos ámbitos, se van haciendo independientes de la supervisión de las personas adultas. Es decir, niños, niñas y adolescentes protagonizan los procesos y no solo son participantes de ellos. Para efectos de este proyecto, se destacan los espacios municipal y de medios de comunicación. El primero de los espacios mencionados es el lugar donde las personas desarrollan gran parte de su vida social, sobre todo niñas y niños que se mantienen en su barrio luego del colegio. 
En complemento al espacio geográfico, el segundo espacio de participación destacado son los medios de comunicación, que aportan en la conformación de una forma de ver la realidad. Estos se transforman en realmente participativos cuando no solo entregan información, sino también ofrecen espacios en que NNA tienen una oportunidad real de opinar, generar contenidos y difundir su forma de mirar el mundo como una forma igual de válida que la de las personas adultas.

Es este cambio, el pasar de ser receptores de un mensaje a ser productores y emisores, el que se puede intencionar en el proceso educomunicativo que se plantea, y el que responde a un escenario comunicativo en que es posible propiciar una cultura de la participación, término planteado por Henry Jenkins que, más allá de una expresión individual, genera una dimensión comunitaria, en que se actúa como usuarios y emisores, potenciando la creatividad y la interacción. Por lo tanto, cualquier propuesta de alfabetización mediática debería estar influenciada por la idea de la cultura de la participación, ya que es necesario desarrollar competencias de recepción y análisis de contenidos, pero también de producción de mensajes y colaboración, entre otras, para generar verdadero protagonismo.

\section{Comunicación y Derechos Humanos}

Para construir una sociedad respetuosa de los Derechos Humanos, se necesitan personas conscientes de sus derechos a nivel individual, pero que además se constituyan en agentes de cambio, y la comunicación es un espacio fértil para ello (Unicef, 2006). Las y los comunicadores pueden aportar desde diferentes dimensiones: ayudar a generar climas de opinión que fomenten cambios en políticas públicas y apoyen transformaciones culturales; promover nuevas capacidades, conductas y valores; difundir información sobre los Derechos Humanos y su influencia en áreas como educación y salud; informar a las personas sobre sus derechos, obligaciones y medidas de exigibilidad, entre otras posibilidades (Unicef, 2006).

Desde esta perspectiva, uno de los objetivos transversales que puede abordar la comunicación es fortalecer la participación, la democracia y la movilización de los sectores sociales por sus demandas comunes. ¿Cómo se puede materializar esto en el trabajo comunicacional? Garantizando el acceso a la información, respetando las diversidades y construyendo nuevas formas de democracia participativa. 
El uso de medios, sobre todo los digitales, contribuye a la creación de identidades sociales y se han convertido en escenarios en que se construye lo público, conociéndose demandas de la sociedad civil. Como comunicadores/as y medios de comunicación se puede asumir un rol de mediación social, construyendo puentes entre la ciudadanía y el Estado; y también un rol formativo y educativo (Unicef, 2006). Rossanna Reguillo señala que la relación entre Derechos Humanos y la comunicación se centra en proyectos socio-políticos diferentes, básicamente es el debate sobre la inclusión o exclusión, que no se limita solo a la integración, sino que remite a las formas de relación entre los seres humanos, en condiciones de respeto mutuo y equidad (Reguillo, 1998).

La comunicación es un espacio privilegiado para la democratización del espacio público. Es, desde esta perspectiva, crear espacios para comunicar, que sean respetuosos de los Derechos Humanos, aporta a la democracia, sobre todo si son niños y niñas de sectores vulnerados quienes tienen la posibilidad de instalar sus voces y miradas en el campo de las comunicaciones, voces que no tienen cabida en el discurso de los medios masivos (Cocq Muñoz, 2014).

Hacer comunicación desde la mirada integral de los Derechos Humanos es una de las formas de evitar vulneraciones, ya que es posible cuestionar los modelos excluyentes presentes en los discursos mediáticos tradicionales y aportar a la construcción de pensamiento crítico.

\section{La radio como potenciador educativo}

En su Manual Urgente para Radialistas Apasionados, José Ignacio López Vigil señala que “lo que los medios sacan a la luz pública, al sonido público, queda valorado. Lo que sale, vale. Y aún más: solo lo que sale, existe” (López Vigil, 2005). Esta situación no es diferente en América Latina, donde la radio ha sido un medio de comunicación que además de informar, entretener y educar, ha tenido una relación directa con la construcción y la defensa de la democracia y los derechos de los sectores más vulnerados (Malbrán, 2010).

En esta zona, más que en otras regiones del mundo, "la sociedad no se ha limitado a la categoría de radioescucha y ha buscado diferentes caminos para llegar a ser radiohablante" (López Vigil, 2007). En la revisión de algunas tendencias de la radio educativa en Latinoamérica, se ve uno de los aportes educativos de este tipo de emisoras: el valor de la palabra propia y pública, más allá del mensaje, ya que el uso de la voz legitima a las personas en su rol social (López Vigil, 2007). Además, la posibilidad de actuar localmente 
es una característica de las radios educativas/ populares que las hace adecuadas para un trabajo con grupos específicos en un territorio delimitado.

Con las posibilidades de transmitir a través de plataformas digitales, se ha flexibilizado la solución a uno de los problemas para instalar y operar una radio: los costos que implica, la necesidad de tener un espacio físico y, lo más complejo, tener un permiso para realizar la actividad de acuerdo a la legislación vigente.

Una radio online se conecta con niños, niñas y adolescentes, que utilizan los medios digitales para comunicarse entre sí, desde computadora, tablet o teléfonos móviles. En un proyecto de radio escolar realizado en Chillán se señala que los y las estudiantes ya conocían estas tecnologías y las manejaban mejor que las personas adultas, pero no las utilizaban necesariamente para investigar, crear contenidos y/o comunicarlos (Ferrada Montecinos, 2010).

La radio online tiene potencialidades que permiten un aumento de las posibilidades de la interacción. Pestano y Rodríguez señalan que es "importante aproximar a la comunidad educativa a ese conjunto de prácticas tecnológicas, no sólo desde la perspectiva de la educación para la recepción, que sin duda es imprescindible, sino también desde la potencialidad productiva, de manera que los ciudadanos, cualquiera que sea su edad, puedan apropiarse convenientemente de los nuevos medios, para que se pueda crear, experimentar y participar de nuevas vías expresivas"( 2004). De esta forma, podemos afirmar que educar para la participación ciudadana exige poner en contacto a los niños y jóvenes con su realidad, con sus experiencias, con la actualidad de la calle y que este encuentro se puede hacer desde y con la radio.

Así, se puede observar que la radio online es una posibilidad cierta y accesible para las comunas y barrios vulnerables, al considerar que requiere de elementos técnicos básicos, que permite producir contenidos locales y distribuirlos a través de un soporte que cada vez es más accesible y usado por niños, niñas y jóvenes, es decir se corresponde con sus usos cotidianos. Además, permite un diálogo que hace unos años habría sido más dificultoso: intercambiar experiencias con radialistas de otros países y extender así los límites de su mundo. 


\section{Tres experiencias de radio para educar en Derechos Humanos en Latinoamérica}

Para hacer una propuesta de taller con fuerte foco en la experiencia, se recogieron tres proyectos para su síntesis, análisis y profundización, relacionadas con los diferentes ejes que se desarrollan en la propuesta de taller para mediadores de radio y Derechos Humanos (Cocq Muñoz, 2014). Cada experiencia profundiza uno de esos ejes y presenta elementos que pueden ser integrados en un nuevo taller, ubicándolos desde las tres dimensiones de la educación en Derechos Humanos: desde, sobre y para. Pero, además, son diferentes acercamientos al trabajo de Derechos Humanos y comunicación, que muestran las posibilidades de abordar el tema respetando la integralidad e indivisibilidad de los derechos.

Las categorías de análisis utilizadas fueron: Los modelos de educación en Derechos Humanos planteados por Tibbitts; la integralidad del concepto de Derechos Humanos utilizado: perspectiva legal y perspectiva ética; y el nivel de participación de las personas involucradas, según la escalera de la participación de Roger Haart.

El primer caso de análisis es Radio Acuarela Online, experiencia desarrollada en la comuna de La Pintana y que se relaciona con el Taller Jóvenes Reporteros de La Pintana. Esta radio comenzó sus transmisiones cuando el facilitador, Jimmy Fernández, pone en marcha un ambicioso proyecto: implementar la radio online. Un punto de apoyo en esta etapa fue la participación en el proyecto "Reporteros: Taller de comunicación para la participación ciudadana activa orientado a niños, niñas y adolescentes de La Pintana (Santiago-Chile)”.

Aprovechando las plataformas digitales gratuitas, el facilitador creó una página de Facebook para visibilizar el proyecto, establecer alianzas y, sobre todo, ampliar el rango de acción de los programas, publicando noticias relacionadas al campo de la niñez, los Derechos Humanos y el movimiento social. Sin embargo, hasta el momento de la presentación de esta propuesta, el espacio en que se promocionan los contenidos de la radio, así como su formato online, son operadas exclusivamente por el mediador, lo que se puede explicar por el tiempo del funcionamiento del proyecto y porque el manejo de redes sociales puede exponer a niñas, niños y jóvenes.

Luego del comienzo de las transmisiones, en octubre de 2013, los programas y el entusiasmo de nuevos equipo ha ido en aumento. Otra de las actividades realizadas ha sido el reporteo en terreno y la cobertura de eventos, como un concierto por los Derechos Humanos y la asistencia a otras actividades de la misma temática. 
El segundo caso es Radio Feroz, experiencia desarrollada en Radio Sur de Argentina, por parte de un grupo de jóvenes de distintos barrios cercanos a la emisora, que asumieron la producción radial como una forma de expresión y para contar lo que pasa en sus comunidades. Esta experiencia fue elegida porque consiguió establecerse como un espacio constante de trabajo, en que los equipos se van renovando. Entre las habilidades que se potencian a través de los talleres, se cuentan "el desarrollo personal y social, promueven la creatividad, el crecimiento de la autoestima, la conciencia crítica y participativa, promoviendo ciudadanía desde las y los más pequeños” (Gerbaldo, 2010). ¿Cómo se propone la formación? Combinando la formación técnica propia del soporte radial y la formación en contenidos de derechos enfocados en niñez y juventud.

Uno de los factores que destaca esta experiencia es que trasciende la formación y la participación en la radio, ya que se han formado monitores en temas de adicciones, enfermedades de transmisión sexual, género, discriminación, entre otros. Al mismo tiempo, se realizan campañas públicas, eventos y actividades presenciales que complementan al programa radial.

El tercer caso es La Radio como una Herramienta para la Construcción de la Paz, proyecto de capacitación que se llevó a cabo durante 2012 en la frontera entre Ecuador y Colombia, a cargo de la Agrupación Bostezo. Este caso fue incluido porque trabajó con radios comunitarias en una zona de violencia y vulneraciones de Derechos Humanos, reuniendo a personas de distintas edades pero con un énfasis en jóvenes. Estos talleres abordaron la temática de los Derechos Humanos como contenido, grabaron 24 cápsulas sobre situaciones cotidianas, pero también se planteó desde los Derechos Humanos, al construir una metodología que respetaba y valoraba las diferencias, tratando de llegar a puntos de acuerdo entre los y las participantes. La forma de trabajo y la profundidad de sus temáticas, el escenario en que se desarrolló, además de su reciente implementación, lo convierten en un modelo interesante para el análisis.

El objetivo del proyecto La Radio Comunitaria como Herramienta para la Construcción de la Paz fue "visibilizar las voces, procesos y experiencias de las organizaciones y las personas vinculadas a las emisoras comunitarias, populares y educativas de ambos lados de la frontera, con especial atención a aquellos procesos que vinculan a organizaciones sociales -en materia de educación, salud, cultura, diversidad, medio ambiente y derechos humanos- con la producción de programas radiofónicos". El taller se estructuró en 
6 momentos o pasos, que combinaron reflexión y producción, usando herramientas radiales simples, ya que el interés estaba centrado en cómo se podían mirar los Derechos Humanos desde la cotidianeidad, reflejando esas historias en la radio

Si bien este proyecto no trabajó directamente con niñez y juventud, fue seleccionado ya que permite observar las tensiones que puede despertar el trabajo con Derechos Humanos, cuando se narra la experiencia de una asistente que al enfrentar el tema de migración, desarrolla un guion radial que considera negativa la llegada de migrantes colombianos a Ecuador. En el relato se señala que esta situación fue resuelta a través de una entrevista de la misma joven a otro colombiano que se encontraba en el taller, disminuyendo sus prejuicios y resistencia al conocer a alguien que rompía sus ideas preconcebidas (Inclán, 2013).

\section{Análisis comparativo de las 3 experiencias radiales}

Radio Acuarela ha pasado, desde sus inicios, por diferentes escalones de participación, llegando al nivel 6. La acción es pensada por agentes externos de desarrollo, pero es compartida con la población, ya que supone que ellos se incorporan en pensar y aportar respecto a la acción a realizar. La temática que aborda Acuarela es el derecho a la participación de niños y jóvenes y a medida que ha avanzado el proyecto, existe mayor autonomía en la producción de los programas radiales. Además, es importante señalar que uno de los objetivos declarados en este proyecto, es tener voces de NNA al aire, en forma exclusiva.

El caso de Radio Feroz, al igual que en el caso anterior, ha pasado por diferentes eslabones para situarse en el nivel 5 de la escalera de la participación, el segundo nivel de participación real. En él, los agentes externos de desarrollo informan y consultan a la población sobre su probable participación y, en base a ello, la población decide. Al igual que el caso anterior, la temática que aborda es el derecho a la participación de niños y jóvenes.

Finalmente, la última experiencia abordada es La Radio como Herramienta para la Paz, que también desarrolla diferentes fases de participación, quedando instalada en el nivel 5. Los agentes externos de desarrollo informan y consultan a la población sobre su probable participación. En base a ello, la población decide. Es un proceso que sigue dependiendo de los agentes externos. A diferencia de las otras experiencias radiales nos encontramos con una temática diferente ya que aborda los Derechos Humanos desde la experiencia cotidiana de la población, trabajando con personas jóvenes y adultas. 
Desde esta perspectiva, todas las experiencias radiales seleccionadas se encuentran en un nivel medio alto de participación en la escalera de Haart. Faltan aún los procesos 6 y 7 , es decir falta aún alcanzar niveles donde la acción se gesta en la propia población y es ejecutada por los actores. Dicho de otra forma, que no esté mediada por los agentes externos de desarrollo. Y un nivel más alto cuando la acción es pensada por la población, pero a diferencia del escalón anterior, es consultada con agentes externos de desarrollo (Cocq Muñoz, 2014).

Para continuar el análisis de las experiencias, se analiza la relación entre los modelos de educación en Derechos Humanos planteados por Tibbitts y las experiencias radiales seleccionadas. En los casos de Radio Acuarela y Radio Feroz se trabajan los Derechos Humanos desde la perspectiva de la participación, y desde ahí se transmite un conocimiento básico para impulsar su integración en los valores públicos. Es un proceso más bien motivacional, pero que no forma en habilidades comunicativas, solución de conflictos y activismo. Ambas consideran el modelo de responsabilidad, ya que esperan que la comunidad se comprometa con la participación. Sin embargo, Radio Feroz es capaz de generar líderes que actúan en la comunidad en diferentes temas, destacándose para el caso de análisis, la problemática de la discriminación que es considerado un tema de Derechos Humanos. Teniendo esto en cuenta, se puede pensar que de alguna forma se asume el modelo de transformación, ya que los programas de educación en Derechos Humanos y formación de capacidades, poniendo el énfasis en la discriminación, están encaminados a facultar al individuo para que reconozca tanto los abusos como para que asuma el compromiso de prevenirlos. En algunos casos, comunidades enteras, no sólo el individuo, constituyen el público a que se espera llegar.

En el caso de La Radio como Herramienta para la Construcción de la Paz el fenómeno es más complejo, ya que incluye el modelo de valores y percepción pero además integra el modelo de responsabilidad, ya que espera que su audiencia asuma un rol en la defensa de los derechos desde sus funciones sociales. Al entender los derechos desde la cotidianidad de la comunidad en una zona de conflicto, esta radio asume el modelo de transformación de conductas, aunque no genere actores capacitados para actuar fuera de la radio en la comunidad.

En el último análisis, señala la relación entre Integralidad del concepto de Derechos Humanos utilizado: perspectiva legal y perspectiva ética y las experiencias radiales en cuestión. 
Tanto Radio Acuarela como Radio Feroz dan énfasis a problemáticas de los derechos de participación, excluyendo otros contenidos legales como sociales, económicos y culturales de los Derechos Humanos y su perspectiva se centra en condiciones éticas más que legales. Es más, los mediadores principales de Radio Acuarela reconocen no tener formación legal en DDHH. La Radio como Herramienta de Construcción para la Paz tiene una gama más amplia en su parrilla temática, sin embargo, su enfoque también es fuertemente ético.

Existe un déficit en las tres experiencias analizadas, en términos de no incluir los diferentes derechos en el ámbito legal, económico, social y cultural, a excepción de la experiencia en la frontera colombo-ecuatoriana y también en solo considerar la perspectiva ética, reduciendo los $\mathrm{DDHH}$ a esta visión, excluyendo la visión legal y restando con ello una perspectiva global de derechos y de posibilitar su exigibilidad.

\section{0. ¿Cómo se plasman los referentes teóricos y el análisis de casos en la propuesta del taller?}

Tomando en cuenta algunos puntos de las tres experiencias analizadas, se propone el diseño del taller Derechos Humanos en el Aire, que tiene como objetivo desarrollar las habilidades comunicativas de NNA y potenciar su empoderamiento como defensores/as de DDHH a través de la radio. Este taller tiene como sello característico que nace desde la experiencia en terreno de un taller anterior y se nutre de aspectos considerados exitosos de las propuestas analizadas (Cocq Muñoz, 2014).

Es un taller pensado como un espacio de aprendizaje de contenidos, actitudes y herramientas radiales que permitan ejercer defensa de derechos a través del ejercicio del derecho a la participación social. ¿Por qué un taller? Porque es un espacio de acción y experiencias concretas, de carácter colectivo, vivencial y donde se comparte con otros y otras. Así, es posible intencionar el diálogo y la convivencia de los distintos puntos de vista.

La propuesta se materializa concretamente en un cuadernillo impreso dirigido a los mediadores de talleres de DDHH en zonas vulnerables, compuesto por actividades que pueden ser utilizadas en forma secuencial o que pueden ser una base para enriquecer el trabajo que ya existe, y un disco compacto que contendrá audios, textos mínimos y textos complementarios para quienes guíen el trabajo. 
Se plantea usar un medio en particular, la radio online, considerando los usos de las y los destinatarios y las posibilidades de este soporte en conjunto con su bajo costo económico. Se proponen estrategias de seguimiento y motivación, considerando la experiencia ya mencionada y tomando algunos puntos destacados de tres experiencias que abarcan al menos dos de estas dimensiones: radio, educación en DDHH y trabajo con niños, niñas y jóvenes.

La figura de los/as mediadores/as se explica por varios factores: la escasa posibilidad de formación en el área de DDHH y radio; la capacidad multiplicadora de estos agentes sociales y la oportunidad de formar diversos grupos a través de liderazgos adultos que los comiencen y luego les entreguen autonomía. El cuadernillo incluye una serie de recomendaciones metodológicas que hay que considerar a la hora de implementar el taller, una lista de temas y actividades. Se pretende que los mediadores utilicen esta guía para incentivar a los niños para que practiquen sus derechos a la participación e incidan sobre las problemáticas de DDHH de su entorno.

Entre los objetivos específicos del taller se encuentran: a) Aprender técnicas para observar y analizar su entorno desde la mirada de los $\mathrm{DDHH}$ integrales; b) Aplicar técnicas periodísticas que le permitan convertirse en productores de mensajes para su comunidad, acordes a sus necesidades e intereses; c) Mejorar su convivencia y capacidades personales a través de una comunicación responsable, creativa y constructiva; d) Compartir y difundir en sus espacios de incidencia local los productos desarrollados como una forma de reivindicar los Derechos Humanos.

Una de las características distintivas del taller es el acompañamiento online, a través de un grupo en redes sociales, como apoyo contra la deserción tan extendida en estas iniciativas y para desarrollar habilidades comunicativas en otros soportes.

El grupo de Facebook, que contaría con la aprobación de padres/madres/apoderados, sería un repositorio de fotografías y audios del grupo (o videos que registren la experiencia), pero también funcionaría como una suerte de foro para acordar desde lo logístico hasta temáticas de trabajo. Es interesante la posibilidad de conectar al grupo con otros grupos radialistas en países distintos, conociendo experiencias y compartiendo inquietudes. Este grupo también tendría una intencionalidad de difusión, como plataforma para dar a conocer las piezas radiales producidas y otros insumos, a la comunidad que el grupo 
intencione. Como se observa, será un componente del taller fundamental a nivel de motivación y de visibilidad.

Otra acción de motivación la constituyen las visitas a medios de comunicación masivos identificables por el grupo. Esta actividad es fundamental para jóvenes que no se identifican con los medios masivos, sino con internet y las redes sociales, pero que están influenciados por los medios y los consumen.

\section{Reflexiones finales}

Los Derechos Humanos sólo pueden hacerse efectivos a través de su vivencia cotidiana y de un conocimiento profundo de sus normas, valores y de su dimensión ética, además de su exigibilidad. De esta forma, es posible que su educación se desarrolle en espacios informales, promoviendo valores éticos, creencias y actitudes que alientan a todas las personas a defender sus propios derechos y los de los demás. Igualmente, desarrolla la conciencia de que todos compartimos la responsabilidad común de hacer de ellos una realidad en todas las comunidades. Cuando son niños, niñas y jóvenes quienes realizan este proceso, la educación en materia de DDHH contribuye de manera esencial a la prevención a largo plazo de abusos y representa una importante inversión en el intento por conseguir una sociedad justa, en la que los derechos de toda persona sean valorados y respetados.

Pero como proceso educativo, es imprescindible considerar la máxima participación de quienes lo viven, además de considerar sus usos cotidianos y sus intereses. Es ahí donde se potencia el trabajo de generar contenidos a través de plataformas cercanas a niños, niñas y jóvenes, como los medios digitales (en este caso la radio online) y las redes sociales, porque en su producción requieren aprender sobre $\mathrm{DDHH}$, pero también vivirlos en el proceso educativo, para que sea una experiencia coherente. A través de las habilidades comunicacionales logran hacer parte a su comunidad más cercana en sus intereses y exigencia de cambios para un mejor vivir.

Las experiencias analizadas respecto a radio y Derechos Humanos muestran diversas formas de abordar este desafío, no exento de dificultades. Utilizando el modelo de transformación, donde niños, niñas y jóvenes identifiquen, denuncien, y con ello sean capaz de prevenir dichas vulneraciones, a través de un medio eficaz como la producción radial en formato digital, que se adapta a sus usos comunicativos, genera en la población un impacto y un gesto difícil de medir, pero con consecuencias que pueden ser 
insospechadas. Niños, niñas y jóvenes sufren un proceso de transformación en su rol e impactan a la comunidad.

Que niños, niñas y jóvenes asuman la responsabilidad de hacer comunicación desde la mirada integral de los DDHH es una de las formas de evitar vulneraciones, ya que es posible cuestionar los modelos excluyentes presentes en los discursos mediáticos tradicionales y aportar a la construcción de pensamiento crítico.

\section{Referencias}

Cocq, P. (2014) Derechos humanos en el aire: diseño de cuadernillo para mediadores de taller en derechos humanos y habilidades comunicativas orientado a niños y jóvenes vulnerables usando la radio. Proyecto para optar al grado de Magíster en Comunicación y Educación, Pontificia Universidad Católica de Chile, Santiago.

Ferrada, Y. e. (2010). Radio escolar online, exploración en informática educativa. Razón y Palabra(74).

Formisano, M. (2011). Empoderando a las personas pobres. País Vasco: Unesco.

Gerbaldo, J. (s/f). Manual de Radio Participativa con niñas, niños y jóvenes Radio Feroz. Córdoba: Ediciones Cecopal.

Gómez, F. (2013). Buenas tardes a todos los vecinos de La Pintana. La experiencia del Taller de Reporteros con adolescentes de la Población San Ricardo.Proyectopara optar al grado de Magíster en Comunicación y Educación, Pontificia Universidad Católica de Chile, Santiago.

Inclán, P. (2013). Desde la frontera colombo-ecuatoriana. (P. Inclán, Ed.)

Instituto Nacional de Derechos Humanos. (2012). Ideaspara introducir los derechos humanos en el aula. Santiago.

Jenkins, H. (2008). La cultura de la convergencia. Barcelona: Paidós.

López, J. (2005). Manual urgente para radialistas apasionados. Quito.

López, J. (noviembre de 2007). La radio latinoamericana: seis aportes al desarrollo. Envío (308).

Malbrán, C. (2010). La radio como herramienta pedagógica. Buenos Aires: Ediciones Corregidor.

Naciones Unidas. (2012). Declaración de las Naciones Unidas sobre educación y formación en materia de derechos humanos. Recuperado el 20 de noviembre de 2013, de Naciones Unidas: http://daccessdds-ny.un.org/doc/UNDOC/GEN/N11/467/07/PDF/N1146707.pdf?OpenElement

Naciones Unidas. (2012). Declaración Universal. Recuperado el 20 de noviembre de 2013, de Naciones Unidas: http://www.un.org/es/documents/udhr/

Nomura, B. y Solari, G. (2005). Participación de niños, niñas y adolescentes a los 15 años de la Convención sobre los Derechos del Niño. Lima: Save the Children Suecia (Programa Regional para América Latina y El Caribe). 
COMUNICACIÓN Y MEDIOS NNo 31 (2015). Instituto de la Comunicación e Imagen, ICEl, Universidad de Chile. ISSN 0719-1529

Oficina del Alto Comisionado de las Naciones Unidas para los Derechos Humanos. (s.f.). Cómo evaluar las actividades de capacitación en derechos humanos. Serie de capacitación profesional №18.

Pestano, J. y Gabino, M. (2004). La Radioweb, instrumento para la innovación educativa. Espéculo. Revista de Estudios Literarios(26).

Sen, A. (2000) La Pobreza como privación de capacidades, en Desarrollo y Libertad.Buenos Aires: Editorial Planeta.

Tibbitts, F. (s/f) Nuevos modelos de educación en derechos humanos. Dehuidela, 15, 75-80. 